\title{
几种著名古陶瓷密遗址粘土的 穆斯堡尔谱研究*
}

高正耀 陈松华 潘贤家

（郑州大学,郑州 450052）
李 国 栋

(中国科学院物理研究所, 北京 100080)

关链词 穆斯堡尔橧、粘土、烧制条件

穆斯堡尔效应具有追踪粘土烧制过程中铁的相变的能力 ${ }^{[1,2]}$. 详细研究粘土烧制过程中 穆斯堡尔谱参数随烧制温度、烧制时间和烧制气氛的变化规律, 对于利用穆斯堡尔谱进行古陶 瓷研究和现代仿古陶瓷的生产有重要意义.

\section{一、实验方法与结果}

样品: $\mathrm{C} 1$ 是俩山粘土, 被认为是制造秦始皇兵马俑的原料 ${ }^{(3)} . \mathrm{C} 2{ }^{* *}$ 是取自奻瓷官密遗址

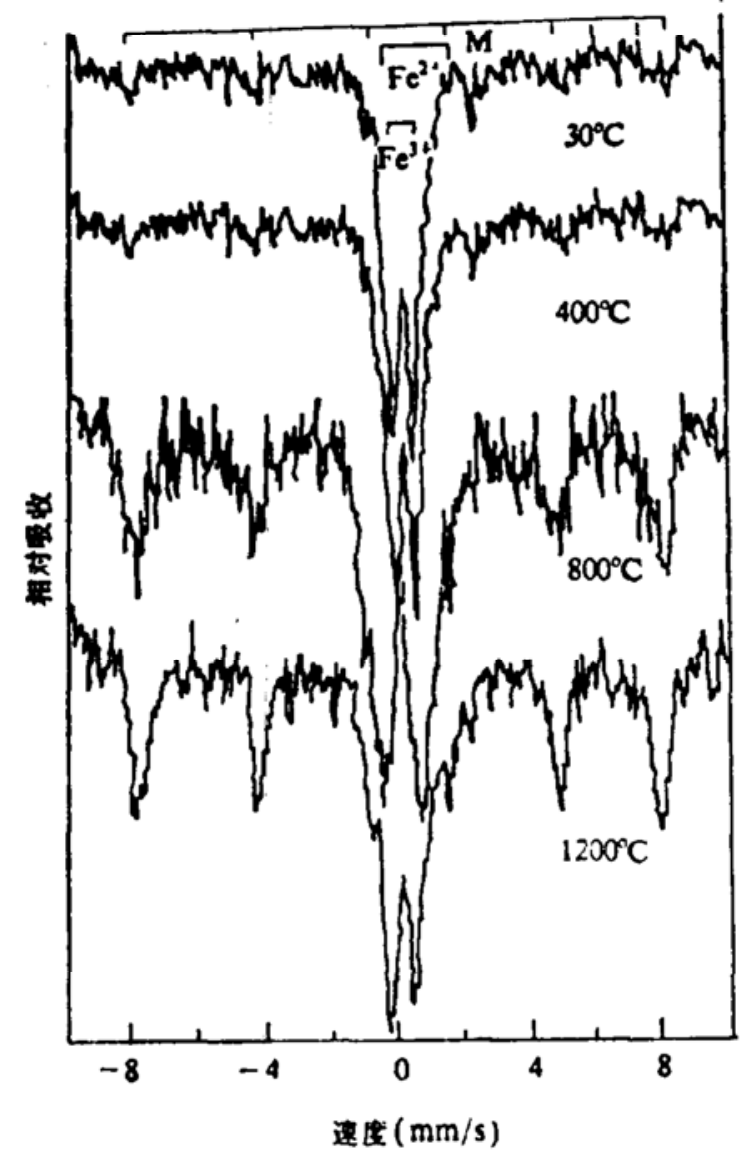

图 1 样品 $\mathrm{C}_{1}$ 在不同烧制温度(烧制 $8 \mathrm{~h}$ )

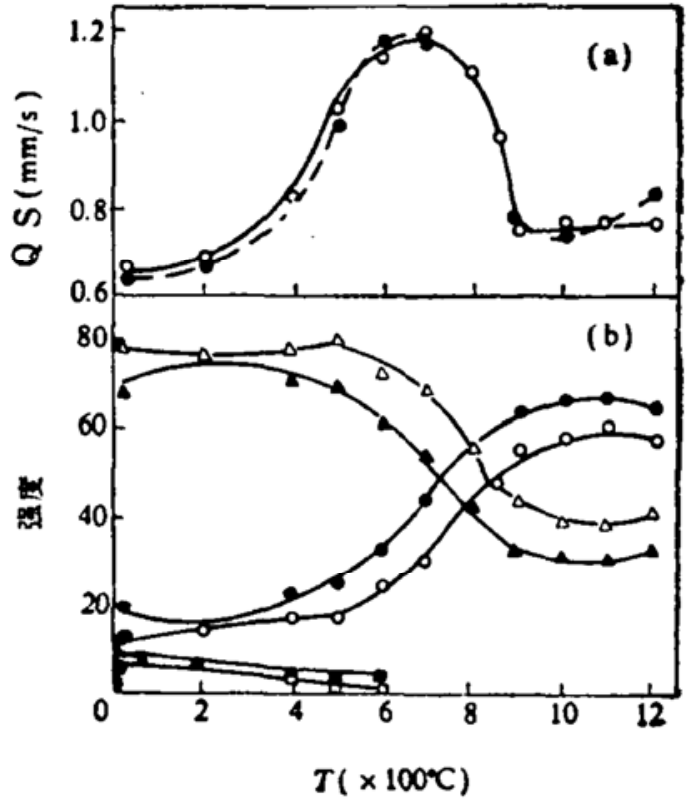

图 2

(2) 样品 $\mathrm{C} 1$ 和 $\mathrm{C2}$ 在不同烧制温度的 $\mathrm{Fe}^{3+} \mathrm{Q} \mathrm{S}$ 值 (烧制 $8 \mathrm{~h}$ ); (b) 样品 $\mathrm{C1}$ 和 $\mathrm{C2}$ 在不同温烧制 温度的 $\mathrm{Fe}^{3+} 、 \mathrm{Fe}^{2+}$ 和 $\mathrm{M}$ 的强度 (烧制 $8 \mathrm{~h}$ ).
(a) $\circ \mathrm{Cl}, \bullet \mathrm{C} 2$;
(b) $\mathrm{M}, \mathrm{Fe}^{2+}, \mathrm{Fe}^{2+}$. $\mathrm{Cl} \bigcirc \Delta \mathrm{B}, \mathrm{C} 2 \bullet \Delta \cdot$ 的室湜穆斯堡尔谱

本文 1989 年 10 月 10 日收到. 1989 年 12 月 25 日收到修改稿.

*国家自然科学基金盗助项目. ** C2 的数据表删诚. 
表 1 样品 $\mathrm{C}_{1}, \mathrm{C}_{2}, \mathrm{C}_{3}, \mathrm{C}_{4}$ 的室温穆斯堡尔谱(未烧)

\begin{tabular}{|c|c|c|c|c|c|c|c|c|c|c|}
\hline \multirow[t]{2}{*}{ 样 品 } & \multicolumn{3}{|c|}{$\mathrm{Fe}^{3+}$} & \multicolumn{3}{|c|}{$\mathrm{Fe}^{2+}$} & \multicolumn{4}{|c|}{$\mathbf{M}$} \\
\hline & $\underset{(\mathrm{mm} / \mathrm{s})}{\mathrm{I} \mathrm{S}}$ & $\underset{(\mathrm{mm} / \mathrm{s})}{\mathrm{Q} \mathrm{S}}$ & $\begin{array}{c}1 \\
(\%)\end{array}$ & $\underset{(\mathrm{mm} / \mathrm{s})}{\text { I S }}$ & $\underset{(\mathrm{mm} / \mathrm{s})}{\text { Q S }}$ & $\stackrel{I}{(\%)}$ & $\underset{(\mathrm{mm} / \mathrm{s})}{1 \mathrm{~s}}$ & $\begin{array}{c}\text { Q S } \\
(\mathrm{mm} / \mathrm{s})\end{array}$ & $\begin{array}{c}\boldsymbol{H} \\
\mathbf{k}[(1000 / \\
4 \pi) \mathrm{A} / \\
\mathrm{m}] \\
\end{array}$ & $\stackrel{I}{(\%)}$ \\
\hline $\mathrm{Cl}$ & 0.345 & 0.666 & 79.7 & 1.055 & 2.876 & 7.2 & 0.376 & -0.298 & 499 & 13.1 \\
\hline $\mathrm{C}_{2}$ & 0.341 & 0.648 & 68.1 & 1.064 & 2.731 & 11.4 & 0.315 & -0.112 & 506 & 20.5 \\
\hline $\mathrm{C} 3$ & 0.327 & 0.669 & 60.0 & 1.031 & 2.708 & 28.9 & 0.333 & -0.166 & 498 & 11.1 \\
\hline $\mathrm{C} 4$ & 0.297 & 0.670 & 57.5 & 1.021 & 2.752 & 24.8 & 0.350 & -0.171 & 508 & 17.7 \\
\hline
\end{tabular}

表 2 样品 $\mathbf{C}_{1}$ 相对于烧制温度和烧制时间的室温穆斯堡尔谱参数

\begin{tabular}{|c|c|c|c|c|c|c|c|c|c|c|c|}
\hline \multirow{2}{*}{$\frac{T}{\mathscr{T}}$} & \multirow{2}{*}{$\frac{t}{\mathrm{~b}}$} & \multicolumn{3}{|c|}{$\mathrm{Fe}^{3+}$} & \multicolumn{3}{|c|}{$\mathrm{Fe}^{2+}$} & \multicolumn{4}{|c|}{$\mathbf{M}$} \\
\hline & & $\underset{(\mathrm{mm} / \mathrm{s})}{\text { I S }}$ & $\begin{array}{c}\mathrm{QS} \\
(\mathrm{mm} / \mathrm{s})\end{array}$ & $\stackrel{1}{(\%)}$ & $\underset{(\mathrm{mm} / \mathrm{s})}{I \mathrm{~S}}$ & $\underset{(\mathrm{mm} / \mathrm{s})}{\mathrm{Q} \mathrm{S}}$ & $\stackrel{I}{(\%)}$ & $\underset{(\mathrm{mm} / \mathrm{s})}{\operatorname{IS}}$ & $\underset{(\mathrm{mm} / \mathrm{s})}{\mathrm{Q} \mathrm{S}}$ & $\begin{array}{r}H \\
\mathbf{k}[(1000 / \\
4 \pi) \mathrm{A} / \\
\mathrm{m}]\end{array}$ & $\stackrel{1}{(\%)}$ \\
\hline 200 & \multirow{11}{*}{8} & 0.297 & 0.697 & 77.0 & 0.968 & 2.733 & 7.9 & 0.303 & -0.255 & 500 & 15.0 \\
\hline 400 & & 0.346 & 0.835 & 78.2 & 0.900 & 2.552 & 3.9 & 0.343 & -0.125 & 497 & 17.9 \\
\hline 500 & & 0.372 & 1.034 & 80.7 & 0.826 & 2.560 & 1.9 & 0.421 & -0.289 & 501 & 17.4 \\
\hline 600 & & 0.342 & 1.148 & 73.7 & 0.563 & 2.399 & 2.1 & 0.354 & -0.266 & 500 & 24.1 \\
\hline 700 & & 0.382 & 1.208 & 68.9 & & & & 0.302 & -0.270 & 507 & 31.0 \\
\hline 800 & & 0.276 & 1.120 & 55.5 & & & & 0.283 & -0.144 & 495 & 44.5 \\
\hline 850 & & 0.320 & 0.963 & 48.8 & & & & 0.354 & -0.213 & 500 & 51.2 \\
\hline 900 & & 0.201 & 0.756 & 44.4 & & & & 0.284 & -0.251 & 499 & 55.6 \\
\hline 1000 & & 0.302 & 0.766 & 40.3 & & & & 0.390 & -0.191 & 494 & 57.9 \\
\hline 1100 & & 0.271 & 0.780 & 38.5 & & & & 0.348 & -0.203 & 499 & 61.5 \\
\hline 1200 & & 0.294 & 0.768 & 41.9 & & & & 0.361 & -0.228 & 487 & 58.1 \\
\hline \multirow{5}{*}{400} & 1 & 0.368 & 0.818 & 73.7 & 0.868 & 2.534 & 4.3 & 0.420 & -0.126 & 511 & 22.0 \\
\hline & 4 & 0.352 & 0.812 & 79.7 & 0.895 & 2.666 & 2.9 & 0.371 & -2.213 & 501 & 17.4 \\
\hline & 8 & 0.346 & 0.835 & 78.2 & 0.900 & 2.552 & 3.9 & 0.343 & -0.125 & 497 & 17.9 \\
\hline & 12 & 0.397 & 0.876 & 75.3 & 1.066 & 2.997 & 1.7 & 0.399 & -0.139 & 508 & 23.0 \\
\hline & 16 & 0.350 & 0.833 & 75.0 & 0.547 & 2.120 & 1.4 & 0.357 & -0.253 & 501 & 23.6 \\
\hline \multirow{5}{*}{600} & 1 & 0.344 & 1.095 & 71.5 & 0.651 & 2.494 & 2.7 & 0.324 & -0.294 & 499 & 25.9 \\
\hline & 4 & 0.356 & 1.130 & 73.0 & 0.477 & 2.399 & 2.7 & 0.332 & -0.230 & 502 & 24.3 \\
\hline & 8 & 0.342 & 1.148 & 73.7 & 0.563 & 2.399 & 2.1 & 0.354 & -0.266 & 500 & 24.2 \\
\hline & 12 & 0.340 & 1.186 & 68.2 & 0.435 & 2.267 & 3.8 & 0.385 & -0.304 & 501 & 27.9 \\
\hline & 16 & 0.359 & 1.133 & 69.0 & 0.610 & 2.827 & 1.2 & 0.362 & -0.274 & 506 & 29.8 \\
\hline \multirow{5}{*}{800} & 1 & 0.265 & 1.129 & 60.6 & & & & 0.292 & -0.214 & 494 & 39.6 \\
\hline & 4 & 0.295 & 1.152 & 59.9 & & & & 0.331 & -0.223 & 501 & 40.1 \\
\hline & 8 & 0.276 & 1.120 & 55.5 & & & & 0.283 & -0.144 & 495 & 44.5 \\
\hline & 12 & 0.254 & 1.132 & 53.6 & & & & 0.304 & -0.257 & 499 & 46.4 \\
\hline & 16 & 0.308 & 1.120 & 53.8 & & & & 0.363 & -0.257 & 496 & 46.2 \\
\hline \multirow{5}{*}{1000} & 1 & 0.273 & 0.766 & 42.3 & & & & 0.354 & -0.207 & 501 & 57.7 \\
\hline & 4 & 0.215 & 0.766 & 40.3 & & & & 0.290 & -0.226 & 496 & 59.7 \\
\hline & 8 & 0.302 & 0.749 & 42.1 & & & & 0.390 & -0.191 & 494 & 57.9 \\
\hline & 12 & 0.369 & 0.763 & 39.5 & & & & 0.452 & -0.213 & 496 & 60.5 \\
\hline & 16 & 0.203 & 0.764 & 37.3 & & & & 0.296 & -0.188 & 496 & 62.7 \\
\hline
\end{tabular}


的河南省宝丰县清凉寺粘土. C3 是取自唐三彩密遗址附近的河南省巩县小黄冶粘土. C4 是 取自唐三彩密遗址附近的河南省巩县大黄冶粘土. 把样品放在电炉里以不同的温度和不同的 时间在氧化条件下 (在空气中) 进行烧制. 将烧制的粘土研碎, 压成 $100 \mathrm{mg} / \mathrm{cm}^{2}$ 的薄片作为 测试样品. 测量室温透射谱. 用计算机对谱线按洛仑兹函数最小二乘法拟合. 用 $\alpha-\mathrm{Fe}$ 作为 速度标定.

1. 未娆粘土的橧形 未烧粘土的谱形明显地由三部分组成: $\mathrm{Fe}^{3+}$ 双峰、 $\mathrm{Fe}^{2+}$ 双盽和 磁性六线峰 (M) (见表 1 和图 1). 不同样品的 $\mathrm{Fe}^{3+}$ 和 $\mathrm{Fe}^{2+}$ 的同质异能移 (IS)、四极分裂 (QS)及内磁场强度 $(H)$ 基本相同. 这三部分的强度的平均值的比为 $\mathrm{Fe}^{3+}: \mathrm{Fe}^{2+}: \mathrm{M}=66: 18: 16$.

2. $\mathrm{Fe}^{3+} \mathrm{QS}$ 值随㛡制温度的变化 样品的 $\mathrm{Fe}^{3+}$ 的 $\mathrm{QS}$ 值是温度的函数 (见表 2). 图 2 (a) 表明变化规律稳定, 两条曲线几乎重合 ${ }^{\omega}$. 温度从 $200-700^{\circ} \mathrm{C}$, QS 值变化为 $0.7-1.2$ $\mathrm{mm} / \mathrm{s}$; 温度从 $700-900^{\circ} \mathrm{C}$, QS 值为 $1.20-0.75 \mathrm{~mm} / \mathrm{s} ; 900-1200^{\circ} \mathrm{C}$, QS 值变化缓慢.

3. 强度随烧制温度的变化 图 2(b) 表示样品 $\mathrm{C} 1$ 和 $\mathrm{C} 2$ 的 $\mathrm{M} 、 \mathrm{Fe}^{3+}$ 和 $\mathrm{Fe}^{2+}$ 的强度随 烧制温度的变化规律 (见表 2). 随温度的升高, $\mathrm{M}$ 的强度增加, $\mathrm{Fe}^{3+}$ 和 $\mathrm{Fe}^{2+}$ 的强度减少. 在 $300^{\circ} \mathrm{C}$ 以前, 强度基本上不变化. $300-900^{\circ} \mathrm{C}$, 强度变化很快, 磁性峰强度迅速上开, $\mathrm{Fe}^{3+}$ 强 度迅速下降. $600{ }^{\circ} \mathrm{C}$ 时 $\mathrm{Fe}^{2+}$ 强度几乎减少到零. $900-1200^{\circ} \mathrm{C}, \mathrm{M}$ 和 $\mathrm{Fe}^{3+}$ 的强度变化缓慢.

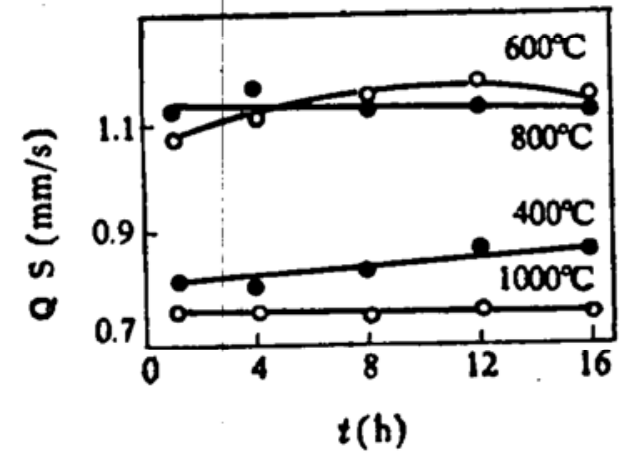

图 3 样品 $\mathrm{C}_{1}$ 在不同烧制温度的 $\mathrm{Fe}^{3+} \mathrm{QS}$ 值 与烧制时间的关系

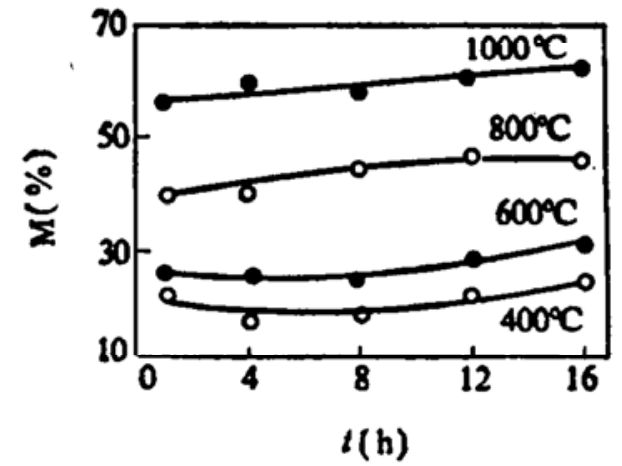

图 4 样品 $\mathrm{Cl}$ 在不同烧制温度的 $\mathrm{M}$ 强度与 烧制时间的关系

不同粘土的变化规律相同, 但与同一温度对应的强度并不相等.

4. 与烧制时间的关系 图 3 表示 $\mathrm{Cl}$ 在不同烧制温度的 $\mathrm{Fe}^{3+} \mathrm{QS}$ 值与烧制时间的 关系. 图 4 表示 $\mathrm{C} 1$ 在不同烧制温度的 $\mathrm{M}$ 强度与烧制时间的关系. $\mathrm{Fe}^{3+} \mathrm{QS}$ 值和 $\mathrm{M}$ 强度随烧制 时间变化不大, 烧制 $8 \mathrm{~h}$ 以后, 特别是温度高于 $400^{\circ} \mathrm{C}$ 时, $\mathrm{Fe}^{3+}$ 的 $\mathrm{QS}$ 值和 $\mathrm{M}$ 强度基本不变.

\section{二、分析与讨论}

1. 未烧粘土 在末烧粘土中存在各种铁矿物, 如赤铁矿、磁铁矿、褐铁矿、纤铁矿、菱铁 矿以及 $\mathrm{FeS}_{2} 、 \mathrm{Fe}_{2}\left(\mathrm{SO}_{4}\right)_{3}$ 等. 这些铁的氧化物和氢氧化物的颗粒大小通常为 $100 \AA$ 的量级. 这 时, 这些细小的颗粒可产生超顺磁双峰. 有些铁以三价铁离子和二价铁离子的形式结合到粘土 矿中, 产生 $\mathrm{Fe}^{3+}$ 和 $\mathrm{Fe}^{2+}$ 顺磁双峰. 我们称这种铁离子为结构铁. 所以在 $\mathrm{Fe}^{3+}$ 双峰中既有氧化 铁小颗粒的贡献,也有结构铁的贡献. 比较粘土的室温谱和液氦温度谱可以区分这两种谱 ${ }^{[2]}$.

2. 娆制㪗土的物理化学变化和稳斯但尔橧特征 现将烧制过程中各阶段的物理、化学 变化和穆斯堡尔谱的特征见表 3. 
表 3

\begin{tabular}{|c|c|c|}
\hline 温 & 物理化学变化 & 楆斯堡尔橧特征 \\
\hline 室温- $300^{\circ} \mathrm{C}$ & 排出坯体残余水分 & 各参数变化甚微 \\
\hline $300-9000^{\circ} \mathrm{C}$ & 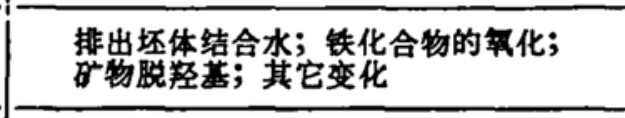 & 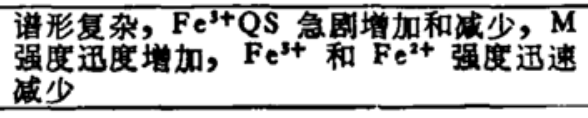 \\
\hline $900-1200{ }^{\circ} \mathrm{C}$ & 形成液相及溶解固体, 形成新结晶, 其他变化 & 谱形简单；数变化甚微 \\
\hline
\end{tabular}

$300-900^{\circ} \mathrm{C}$ 是重要而复杂的阶段,影响穆斯堡尔参数的因素很多:

(1) 排出粘土矿的结合水.

（2）粘土中的所有铁矿物都发生相变. 如:

$300-450^{\circ} \mathrm{C}, \quad \mathrm{FeS}_{2}+\mathrm{O}_{2} \longrightarrow \mathrm{FeS}+\mathrm{SO}_{2} ; \quad 500-800^{\circ} \mathrm{C}, \quad 4 \mathrm{FeS}+7 \mathrm{O}_{2} \longrightarrow 2 \mathrm{Fe}_{2} \mathrm{O}_{3}+$ $4 \mathrm{SO}_{2} ; 400{ }^{\circ} \mathrm{C}$. 以上, $2 \mathrm{FeOOH} \longrightarrow \mathrm{Fe}_{2} \mathrm{O}_{3}+\mathrm{H}_{2} \mathrm{O} ; 400{ }^{\circ} \mathrm{C}$ 以上, $\gamma-\mathrm{Fe}_{2} \mathrm{O}_{3} \longrightarrow \alpha-$ $\mathrm{Fe}_{2} \mathrm{O}_{3} ; \quad 560-7500^{\circ} \mathrm{C}, \quad \mathrm{Fe}_{2}\left(\mathrm{SO}_{4}\right)_{3} \longrightarrow \mathrm{Fe}_{2} \mathrm{O}_{3}+3 \mathrm{SO}_{3} ; 8000^{\circ} \mathrm{C}$ 以上, $4 \mathrm{FeCO}_{3}+$ $\mathrm{O}_{2} \longrightarrow 2 \mathrm{Fe}_{2} \mathrm{O}_{3}+4 \mathrm{CO}_{2}$.

从 $400{ }^{\circ} \mathrm{C}$ 开始, 随着温度的增加各种铁矿物逐步变成赤铁矿. 同时, 二价结构铁氧化成 三价铁. 这些赤铁矿粒子渐渐变大. 所以, 由 $\mathrm{Fe}^{3+}$ 和 $\mathrm{Fe}^{2+}$ 双峰的强度减弱和磁性六线峰 $\mathrm{M}$ 的增强, 可以探测到这种变化. 当温度达到 $800^{\circ} \mathrm{C}$ 以上时各种铁矿物都转变成 $a-\mathrm{Fe}_{2} \mathrm{O}_{3}, \alpha-$ $\mathrm{Fe}_{2} \mathrm{O}_{3}$ 中的铁原子的外电子壳层为 $3 d^{3}$, 是半满壳层状态,具有比较稳定的结构. $900-1250^{\circ} \mathrm{C}$ 之间, $\alpha-\mathrm{Fe}_{2} \mathrm{O}_{3}$ 是唯一存在的铁矿物形式,出现纯 $\alpha-\mathrm{Fe}_{2} \mathrm{O}_{3}$ 谱. 所以穆斯堡尔谱形简单, $\mathrm{Fe}^{3+}$ 和 $\mathrm{M}$ 的强度变化缓慢.

（3）各种粘土矿物在此温区发生脱羟基过程: $\mathrm{OH}^{-}+\mathrm{OH}^{-} \longrightarrow \mathrm{H}_{2} \mathrm{O}+\mathrm{O}^{2-}$.

粘土矿晶格失去结构羟基. 如高岭石脱羟基后, 导致原来八面体中的置换铁原子(六重对 称)处于五重对称珄.铁原子核处电场梯度增加. 由三价铁四极分裂值明显增加到 $1.2 \mathrm{~mm} / \mathrm{s}$ 以 上,可以清楚地观察到这种变化. 在 $800{ }^{\circ} \mathrm{C}$ 以上 $\mathrm{QS}$ 值迅速减小, 这是粘土晶格破坏引起的.

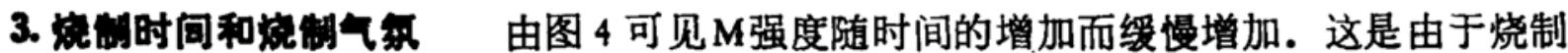
时间越长铁的相变越充分. 穆斯堡尔参数受烧制时间的影响不显著. 特别是在烧制 $8 \mathrm{~h}$ 之后, 参数比较稳定. 这对利用穆斯堡尔谱研究古陶瓷是有意义的. 比较古陶瓷和未烧粘土中的 $\mathrm{Fe}^{3+} 、 \mathrm{Fe}^{2+}$ 和 $\mathrm{M}$ 的强度, 可以判断古陶器的烧成气氛和来源. 穆斯堡尔效应作为研究古陶瓷 的一种手段具有独特的优势.

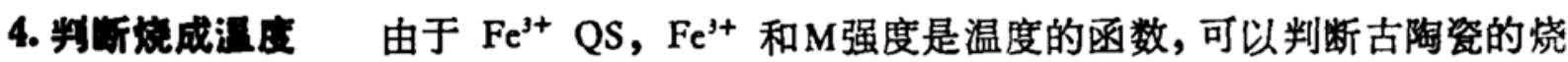
成温度. 在 $700{ }^{\circ} \mathrm{C}$ 附近, $\mathrm{Fe}^{3+} \mathrm{QS}$ 的变化比较缓慢, 因此,不可能达到很高的精度. 在此温度 范围, $\mathrm{Fe}^{3+}$ 和 $\mathrm{M}$ 的强度是温度的单调函数, 用来判断温度更有希望. 高于 $900{ }^{\circ} \mathrm{C}$ 穆斯堡尔参 数变化缓慢, 要准确判断烧制温度应和其他方法一起使用, 如 X 射线衍射分析、岩相学和矿物 学分析等, 才能得到较高的精度.

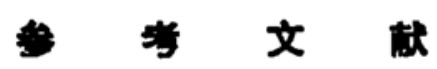

[1] Stevens, J. G., Zhu Wenjun, Hyperjine Intcractions, 29(1986), 1149.

[2] Bouchez, R., Coey, J. M. D. et al., J. Phys., 35(1974), C6-541.

[3] Sun Zhongtian, Pan Xisnjia and Qin Guangyong, Hyperfine Interactions, 11(1988), 847--850.

[4] Gangas, N. H. et al., Clays and Clay Minerals, 21 (1973), 151-160. 